\title{
GEOMETRIC DESCRIPTION OF THE CLASSIFICATION OF HOLOMORPHIC SEMIGROUPS
}

\author{
DIMITRIOS BETSAKOS
}

(Communicated by Jeremy Tyson)

\begin{abstract}
We consider parabolic semigroups $\left(\phi_{t}\right)_{t \geq 0}$ of holomorphic selfmaps of the unit disk $\mathbb{D}$ with Denjoy-Wolff point 1 , Koenigs function $h$ and associated planar domain $\Omega$. We give a geometric description of the classification of such semigroups: The semigroup is of positive hyperbolic step if and only if $\Omega$ is contained in a horizontal half-plane. Moreover, a semigroup of positive hyperbolic step has trajectories that converge to 1 strongly tangentially (namely the semigroup is of finite shift) if and only if $h$ is conformal at 1 .
\end{abstract}

\section{INTRODUCTION}

A one-parameter continuous semigroup of holomorphic functions in the unit disk $\mathbb{D}$ is a family of holomorphic functions $\phi_{t}: \mathbb{D} \rightarrow \mathbb{D}, t \geq 0$, with the properties:

(a) $\phi_{0}$ is the identity in $\mathbb{D}$.

(b) $\phi_{t+s}=\phi_{t} \circ \phi_{s}$, for all $t, s \geq 0$.

(c) $\lim _{t \rightarrow s} \phi_{t}(z)=\phi_{s}(z)$, for all $s \geq 0$ and all $z \in \mathbb{D}$.

The fundamental results of the theory of such semigroups are presented in [1, Chapter 1.4]. This book contains also detailed references to the original contributions. The main trends of the current research on the subject may be found in [4], 12, [13, 15], 16].

The basic characteristic of the dynamical behavior of a semigroup $\left(\phi_{t}\right)$ is described as follows (see [1, Theorem 1.4.17]): There exists a point $\tau \in \mathbb{D} \cup \partial \mathbb{D}$ such that for every $z \in \mathbb{D}$,

$$
\lim _{t \rightarrow+\infty} \phi_{t}(z)=\tau \text {. }
$$

This point $\tau$ is called the Denjoy-Wolff point of the semigroup. The semigroups with interior Denjoy-Wolff point are called elliptic. In the present article we will be interested in semigroups with boundary Denjoy-Wolff points. In this case we may assume without essential loss of generality that the Denjoy-Wolff point is the point 1. This point is the unique attractive boundary fixed point of each of the functions $\phi_{t}, t \geq 0$. This means that for every $t \geq 0$,

$$
\angle \lim _{z \rightarrow 1} \phi_{t}(z)=1 \quad \text { (angular limit) }
$$

Received by the editors December 2, 2014 and, in revised form, April 20, 2015.

2010 Mathematics Subject Classification. Primary 30D05, 37L05, 30C45.

Key words and phrases. Semigroup of holomorphic functions, Denjoy-Wolff point, Koenigs function, parabolic semigroup, Abel equation, positive hyperbolic step, finite shift, angular derivative. 
and

$$
\phi_{t}^{\prime}(1):=\angle \lim _{z \rightarrow 1} \frac{\phi_{t}(z)-1}{z-1} \leq 1 \quad \text { (angular derivative). }
$$

The semigroup is called hyperbolic if for some (equivalently, for all) $t>0$, $\phi_{t}^{\prime}(1)<1$; the semigroup is called parabolic if $\phi_{t}^{\prime}(1)=1$. See [4, Theorem 2.1].

Every semigroup $\left(\phi_{t}\right)$ with Denjoy-Wolff point 1 has a very useful representation [1, Theorem 1.2.22]: There exists a unique conformal mapping $h: \mathbb{D} \rightarrow \mathbb{C}$ such that $h(0)=0$ and

$$
\phi_{t}(z)=h^{-1}(h(z)+t), \quad t \geq 0, z \in \mathbb{D} .
$$

The map $h$ is called the Koenigs function of the semigroup. The domain $\Omega:=h(\mathbb{D})$ is the associated planar domain. It follows from (1.4) that $\Omega$ is convex in the positive direction; this means that if $w \in \Omega$, then the half-line $\{w+s: s \geq 0\}$ is contained in $\Omega$.

By a theorem of Contreras and Díaz-Madrigal [4, Theorem 2.1], we can decide whether a semigroup with Denjoy-Wolff point 1 is hyperbolic or parabolic simply by looking at its associated planar domain $\Omega$ : If $\Omega$ is contained in a horizontal strip, then the semigroup is hyperbolic; otherwise, it is parabolic.

Contreras and Díaz-Madrigal introduced a further distinction within the class of parabolic semigroups. For $w \in \Omega$, they let $\delta_{\Omega}(w)$ be the distance of $w$ from the boundary of $\Omega$ and considered parabolic semigroups for which the limit $\lim _{s \rightarrow+\infty} \delta_{\Omega}(w+s)$ is finite, and parabolic semigroups for which this limit is infinite. They showed that these two classes have different dynamic behavior, when $t \rightarrow+\infty$. We will further clarify this classification of parabolic semigroups.

We say that a parabolic semigroup is of zero hyperbolic step if for some $s>0$ and some $z \in \mathbb{D}$,

$$
\lim _{t \rightarrow+\infty} \rho_{\mathbb{D}}\left(\phi_{t}(z), \phi_{t+s}(z)\right)=0 .
$$

Here $\rho_{\mathbb{D}}$ denotes the hyperbolic distance in $\mathbb{D}$. Note that by the Schwarz-Pick lemma and the semigroup property, the function

$$
[0,+\infty) \ni t \mapsto \rho_{\mathbb{D}}\left(\phi_{t}(z), \phi_{t+s}(z)\right) \in[0,+\infty)
$$

is decreasing. So the limit in (1.6) exists. If (1.5) does not hold for some $s>0$ and some $z \in \mathbb{D}$, then the limit is equal to a positive number (which depends on $s$ and $z$ ). In this case, we say that the semigroup is of positive hyperbolic step.

The above terminology comes from the theory of iteration of holomorphic selfmaps of $\mathbb{D}$. The seminal papers of that theory are [2] and 20]. We also refer to [1], [8], 9], 11], 13], 17, [18, 19], 22. Let $\phi$ be a holomorphic self-map of $\mathbb{D}$ with no fixed point in $\mathbb{D}$. By the Denjoy-Wolff theorem the $n$-th iterate $\phi_{n}=\phi \circ \cdots \circ \phi$ ( $n$ times) converges (as $n \rightarrow+\infty$ ) uniformly on compact subsets of $\mathbb{D}$ to a point $\tau \in \partial \mathbb{D}$ which is called the Denjoy-Wolff point of $\phi$. Moreover, $\phi$ has finite nonzero angular derivative at $\tau$ and $\phi^{\prime}(\tau) \leq 1$. The function $\phi$ is called hyperbolic if $\phi^{\prime}(\tau)<1$ and parabolic if $\phi^{\prime}(\tau)=1$. A parabolic function $\phi$ is said to be of zero hyperbolic step if for some (equivalently, for all) $z \in \mathbb{D}$,

$$
\lim _{n \rightarrow+\infty} \rho_{\mathbb{D}}\left(\phi_{n}(z), \phi_{n+1}(z)\right)=0 ;
$$

otherwise, $\phi$ is said to be of positive hyperbolic step. Note that if a semigroup $\left(\phi_{t}\right)$ has Denjoy-Wolff 1 and it is parabolic, then each of its member functions $\phi_{t}$ has Denjoy-Wolff 1 and it is parabolic. 
The theorem below shows that the above classification of parabolic semigroups is well defined. Moreover, the theorem provides a simple geometric description of this classification.

Theorem 1. Suppose that $\left(\phi_{t}\right)$ is a parabolic semigroup with Denjoy-Wolff point 1 and associated planar domain $\Omega$. The following are equivalent.

(1) $\left(\phi_{t}\right)$ is of positive hyperbolic step.

(2) For every $z \in \mathbb{D}$ and every $s>0$, the limit $\lim _{t \rightarrow+\infty} \rho_{\mathbb{D}}\left(\phi_{t}(z), \phi_{t+s}(z)\right)$ exists and it is equal to a strictly positive number.

(3) For every $t>0$, the function $\phi_{t}$ is of positive hyperbolic step.

(4) For some $t>0$, the function $\phi_{t}$ is of positive hyperbolic step.

(5) For some $w \in \Omega$, the limit $\lim _{t \rightarrow+\infty} \delta_{\Omega}(w+t)$ exists and is finite.

(6) For every $w \in \Omega$, the limit $\lim _{t \rightarrow+\infty} \delta_{\Omega}(w+t)$ exists and is finite.

(7) The domain $\Omega$ is contained in a horizontal half-plane.

The equivalence of (1) and (7) is implicit in [8].

Of course, Theorem 1 implies a corresponding statement for parabolic semigroups of zero hyperbolic step. We state a shortened version of this statement.

Corollary 1. Suppose that $\left(\phi_{t}\right)$ is a parabolic semigroup with Denjoy-Wolff point 1 and associated planar domain $\Omega$. The following are equivalent.

(1) $\left(\phi_{t}\right)$ is of zero hyperbolic step.

(2) For every $t>0$, the function $\phi_{t}$ is of zero hyperbolic step.

(3) For every $w \in \Omega, \lim _{t \rightarrow+\infty} \delta_{\Omega}(w+t)=+\infty$.

(4) The domain $\Omega$ is not contained in a horizontal half-plane.

The equivalence of (1) and (2) is also proved in [5, Lemma 5.1]

There is a different classification of semigroups that involves the trajectories of the semigroup. Let $\left(\phi_{t}\right)$ be a semigroup. For $z \in \mathbb{D}$, the curve

$$
\gamma_{z}:[0,+\infty) \rightarrow \mathbb{D} \text { with } \gamma_{z}(t)=\phi_{t}(z)
$$

is the trajectory starting from $z$. Note that $\gamma_{z}$ tends asymptotically to 1 as $t \rightarrow+\infty$. An important object of study is the precise description of the asymptotic behavior (rate of convergence, angular characteristics) of the trajectories in relation with the type of the semigroup; see [3], 4], [6], 13], [16] and references therein.

A horodisk at 1 is a disk of the form

$$
\Delta_{\eta}=\{z \in \mathbb{D}: d(z, 1)<\eta\}, \quad 0<\eta<+\infty,
$$

where

$$
d(z, 1)=\frac{|1-z|^{2}}{1-|z|^{2}}, \quad z \in \mathbb{D} .
$$

Note that $\Delta_{\eta}$ is internally tangent to the unit circle at the point 1 . We say that the trajectory $\gamma_{z}$ converges to 1 strongly tangentially if there exists an $\eta>0$ such that (the image of) $\gamma_{z}$ lies outside $\Delta_{\eta}$. We will see later that this property does not depend on the initial point $z$ of the trajectory. Note that by Julia's lemma (see [1] or 22]), for $z \in \mathbb{D}$, the function

$$
(0,+\infty) \ni t \mapsto d\left(\gamma_{z}(t), 1\right) \in(0,+\infty)
$$

is decreasing. Therefore, $\left(\phi_{t}\right)$ has strongly tangential trajectories if the limit

$$
\lim _{t \rightarrow+\infty} d\left(\gamma_{z}(t), 1\right)
$$


is equal to a positive number (which may depend on $z$ ). In this case we say that the semigroup is of finite shift. Otherwise, when the limit is equal to 0 , we say that the semigroup has weakly tangential trajectories, or that the semigroup is of infinite shift. Observe that every trajectory of a semigroup of infinite shift penetrates every sufficiently small horodisk as it approaches the attractive point 1 . Again the terminology comes from the theory of iteration (see [9, p. 66]). A holomorphic function $\phi: \mathbb{D} \rightarrow \mathbb{D}$ is of finite shift if for some (equivalently for all) $z \in \mathbb{D}$,

$$
\lim _{n \rightarrow+\infty} d\left(\phi_{n}(z), 1\right)<+\infty ;
$$

otherwise, when the limit is equal to $0, \phi$ is of infinite shift. The important role of these definitions in iteration theory was observed by Poggi-Corradini [17.

If a semigroup is hyperbolic, then for every $z \in \mathbb{D}$ and every $t>0$,

$$
d\left(\phi_{t}(z), 1\right)<e^{-\beta t} d(z, 1)
$$

for some $\beta>0$; see [13, p. 24]. Therefore, the semigroup (and each of its members) is of finite shift. So we concentrate on parabolic semigroups.

Theorem 2. Suppose that $\left(\phi_{t}\right)$ is a parabolic semigroup with Denjoy-Wolff point 1. The following are equivalent.

(1) $\left(\phi_{t}\right)$ is of infinite shift.

(2) For every $z \in \mathbb{D}, \lim _{t \rightarrow+\infty} d\left(\phi_{t}(z), 1\right)=0$.

(3) For every $t>0$, the function $\phi_{t}$ is of infinite shift.

(4) For some $t>0$, the function $\phi_{t}$ is of infinite shift.

Corollary 2. Suppose that $\left(\phi_{t}\right)$ is a parabolic semigroup with Denjoy-Wolff point 1. The semigroup is of finite shift if and only if for every $t>0$, the function $\phi_{t}$ is of finite shift.

Suppose now that $\left(\phi_{t}\right)$ is a parabolic semigroup with Denjoy-Wolff point 1 and zero hyperbolic step. By Theorem 1, for every $t>0, \phi_{t}$ is a function of zero hyperbolic step. Then by a theorem of Poggi-Corradini [19, Proposition 4.1] (see also [10, Proposition 3.3]), $\phi_{t}$ is of infinite shift. It follows from Theorem 2 that the semigroup $\left(\phi_{t}\right)$ is of infinite shift. Therefore, we can classify the parabolic semigroups into three disjoint classes:

(a) Semigroups with zero hyperbolic step and infinite shift.

(b) Semigroups with positive hyperbolic step and infinite shift.

(c) Semigroups with positive hyperbolic step and finite shift.

By Theorem 1] if $\Omega$ is not contained in a horizontal half-plane, then the semigroup belongs to class (a). We will give a geometric description for the classes (b) and (c). The description involves the classical notion of conformality of a conformal map $f: \mathbb{D} \rightarrow \mathbb{C}$ at a boundary point $\zeta \in \partial \mathbb{D}$. The pertinent theory is presented in [14] and 21]. We state the definition for the point $\zeta=1$. The function $f$ is called conformal at 1 if the angular limit $f(1) \neq \infty$ exists and the angular derivative $f^{\prime}(1)$ exists and it is finite and non-zero. If $f(1)=\infty$, we consider a Möbius map $\psi$ sending $\infty$ to a finite point and we say that $f$ is conformal at 1 if $\psi \circ f$ is conformal at 1. Also, if $F$ maps a half-plane $\Pi$ conformally into itself and $F(\infty)=\infty$ (angular limit), we consider a Möbius map $\varphi$ sending $\Pi$ onto $\mathbb{D}$ and $\infty$ to 1 and we say that $F$ is conformal at infinity if $\varphi \circ F \circ \varphi^{-1}: \mathbb{D} \rightarrow \mathbb{D}$ is conformal at 1 . 
Theorem 3. Let $\left(\phi_{t}\right)$ be a parabolic semigroup with Denjoy-Wolff point 1 , Koenigs function $h$ and associated planar domain $\Omega$. Suppose that $\left(\phi_{t}\right)$ is of positive hyperbolic step and let

$$
H_{\rho}=\{w \in \mathbb{C}: \operatorname{Im} w>-\rho\}, \quad \rho>0,
$$

be the smallest horizontal half-plane containing $\Omega$. The following are equivalent.

(1) $\left(\phi_{t}\right)$ is of finite shift.

(2) If $\Delta$ is a horodisk at 1 , then $h(\Delta)$ lies in a horizontal half-plane which is strictly contained in $H_{\rho}$.

(3) $h$ is conformal at 1.

The corresponding result for infinite shift is the following:

Corollary 3. Let $\left(\phi_{t}\right)$ be a parabolic semigroup of positive hyperbolic step with Denjoy-Wolff point 1 and Koenigs function $h$. The semigroup $\left(\phi_{t}\right)$ is of infinite shift if and only if $h$ is not conformal at 1.

We note that conformality of $h$ at 1 is a geometric property of $\Omega$. By a theorem of Jenkins, Oikawa, Rodin, and Warschawski (see [14, Chapter V], [21, Chapter $11]$ ), there exists a necessary and sufficient geometric condition (involving extremal length) that guarantees the conformality of $h$ at 1 . In general, this condition is extremely delicate but in many cases it implies simple Euclidean geometric conditions.

If (in the context of Theorem (3) $\Omega$ contains a horizontal half-plane, then its boundary is contained in a strip. Then one can use [21, Theorem 4.14] and conclude that $h$ is conformal at 1 . Thus we obtain a simple condition that implies that the semigroup is of finite shift:

Corollary 4. Let $\left(\phi_{t}\right)$ be a parabolic semigroup of positive hyperbolic step with Denjoy-Wolff point 1 and associated planar domain $\Omega$. If $\Omega$ contains a horizontal half-plane, then $\left(\phi_{t}\right)$ is of finite shift.

\section{Proof of Theorem 1}

The implications $(6) \Rightarrow(5),(2) \Rightarrow(1),(3) \Rightarrow(4),(7) \Rightarrow(6)$ are obvious.

$(5) \Rightarrow(7)$ : We assume that for some $w \in \Omega, \delta_{\Omega}(w+t) \rightarrow \rho<\infty$ as $t \rightarrow+\infty$. Then

$$
\lim _{n \rightarrow+\infty} \delta_{\Omega}(w+n)=\rho .
$$

We claim that for every $n \in \mathbb{N}$, the closed vertical segment

$$
K_{n}=[w+n-i \rho, w+n+i \rho]
$$

intersects the boundary of $\Omega$. Indeed, if $K_{m} \subset \Omega$ for some $m$, then by the convexity of $\Omega$ in the positive direction, the closed half-strip

$$
\{u+i v \in \mathbb{C}: u \geq \operatorname{Re} w+m, \operatorname{Im} w-\rho<v<\operatorname{Im} w+\rho\}
$$

would be contained in $\Omega$. Hence $\delta_{\Omega}(w+n)>\rho$ for every sufficiently large $n$. Since $\delta_{\Omega}(w+n)$ is an increasing sequence, this contradicts (2.1).

Therefore, there exist positive integers $n_{1}<n_{2}<\ldots$ such that each $K_{n_{k}}^{-}$or each $K_{n_{k}}^{+}$intersects $\partial \Omega$, where

$$
K_{n}^{-}=[w+n-i \rho, w+n], \quad K_{n}^{+}=[w+n, w+n+i \rho] .
$$


We assume the first possibility (the second is treated in a similar way). Suppose that there exists $w_{1} \in \Omega$ with $\operatorname{Im} w_{1}<-\rho$. Since $\Omega$ is connected, there is a path $\alpha$ in $\Omega$ joining $w_{1}$ with $w$. Let

$$
s_{1}=\max \{\operatorname{Re} \zeta: \zeta \in \alpha\} .
$$

Take $n_{k}$ large enough so that $\operatorname{Re} w+n_{k}>s_{1}$. Then there exists a point $w+n_{k}-i \eta \in$ $\partial \Omega$ with $0<\eta \leq \rho$. By the convexity of $\Omega$ in the positive direction, the half-line

$$
\left\{w+n_{k}-i \eta+s: s \leq 0\right\}
$$

lies in the complement of $\Omega$ and intersects the path $\alpha \subset \Omega$; a contradiction.

$(7) \Rightarrow(2)$ : Suppose that $\Omega$ is contained in a horizontal half-plane $H$. We denote by $\rho_{\Omega}$ and $\rho_{H}$ the hyperbolic distances in $\Omega$ and $H$. By the conformal invariance and the domain monotonicity of the hyperbolic distance, for $z \in \mathbb{D}, t, s>0$, and $w=h(z)$,

$$
\begin{aligned}
\rho_{\mathbb{D}}\left(\phi_{t}(z), \phi_{t+s}(z)\right) & =\rho_{\Omega}(w+t, w+t+s) \\
& \geq \rho_{H}(w+t, w+t+s) \\
& =\rho_{H}(w, w+s)>0 .
\end{aligned}
$$

Therefore the decreasing function $t \mapsto \rho_{\mathbb{D}}\left(\phi_{t}(z), \phi_{t+s}(z)\right)$ converges to a strictly positive number, as $t \rightarrow+\infty$.

$(1) \Rightarrow(5)$ : Suppose that (1) holds but (5) is false. Then for every $w \in \Omega$,

$$
\lim _{t \rightarrow+\infty} \delta_{\Omega}(w+t)=+\infty \text {. }
$$

Let $z=h^{-1}(w)$ and denote by $\lambda_{\Omega}$ the density of the hyperbolic metric in $\Omega$. By well-known properties of the hyperbolic distance (see [21, §4.6]), for every $s>0$,

$$
\begin{aligned}
\rho_{\mathbb{D}}\left(\phi_{t}(z), \phi_{t+s}(z)\right) & =\rho_{\Omega}(w+t, w+t+s) \\
& \leq \int_{0}^{s} \lambda_{\Omega}(w+t+r) d r \\
& \leq \int_{0}^{s} \frac{1}{\delta_{\Omega}(w+t+r)} d r \\
& \leq \frac{s}{\delta_{\Omega}(w+t)} .
\end{aligned}
$$

It follows from (2.3) and (2.4) that for every $z \in \mathbb{D}$ and every $s>0$,

$$
\lim _{t \rightarrow+\infty} \rho_{\mathbb{D}}\left(\phi_{t}(z), \phi_{t+s}(z)\right)=0 .
$$

This contradicts (1).

$(2) \Rightarrow(3)$ : Let $t>0$ and $z \in \mathbb{D}$. Because of (2), the limit

$$
\lim _{n \rightarrow+\infty} \rho_{\mathbb{D}}\left(\phi_{t n}(z), \phi_{t(n+1)}(z)\right)=\lim _{n \rightarrow+\infty} \rho_{\mathbb{D}}\left(\phi_{t n}(z), \phi_{t n+t}(z)\right)
$$

exists and it is equal to a positive number. Hence the function $\phi_{t}$ is of positive hyperbolic step.

$(4) \Rightarrow(1)$ : Suppose that for some $s>0$, the function $\phi_{s}$ is of positive hyperbolic step. Consider the sequence $t_{n}=n s, n \in \mathbb{N}$. Then for $z \in \mathbb{D}$, the sequence

$$
\rho_{\mathbb{D}}\left(\phi_{t_{n}}(z), \phi_{t_{n}+s}(z)\right)=\rho_{\mathbb{D}}\left(\phi_{s n}(z), \phi_{s(n+1)}(z)\right)
$$

converges to a positive number. Since the function

$$
(0,+\infty) \ni t \mapsto \rho_{\mathbb{D}}\left(\phi_{t}(z), \phi_{t+s}(z)\right) \in(0,+\infty)
$$


is decreasing, we conclude that the limit

$$
\lim _{t \rightarrow+\infty} \rho_{\mathbb{D}}\left(\phi_{t}(z), \phi_{t+s}(z)\right)
$$

is equal to a positive number. This means that the semigroup $\left(\phi_{t}\right)$ is of positive hyperbolic step.

We have proved that $(2) \Rightarrow(3) \Rightarrow(4) \Rightarrow(1) \Rightarrow(5) \Rightarrow(7) \Rightarrow(2)$ and that $(7) \Rightarrow(6) \Rightarrow(5)$. So all the conditions (1)-(7) are equivalent and Theorem 1 has been proved.

\section{Proof of Theorem 2}

The implications $(2) \Rightarrow(1)$ and $(4) \Rightarrow(3)$ are obvious.

$(1) \Rightarrow(4)$ : By $(1)$, for some $z \in \mathbb{D}$,

$$
\lim _{t \rightarrow+\infty} d\left(\phi_{t}(z), 1\right)=0 .
$$

It follows that for this $z$ and any $t>0$,

$$
\lim _{n \rightarrow+\infty} d\left(\phi_{t n}(z), 1\right)=0 .
$$

So the function $\phi_{t}$ is of infinite shift.

$(3) \Rightarrow(2)$ : Suppose that for some $t>0$, the function $\phi_{t}$ is of infinite shift. Then for $z \in \mathbb{D}$,

$$
\lim _{t \rightarrow+\infty} d\left(\phi_{t n}(z), 1\right)=0
$$

But $d\left(\phi_{u}(z), 1\right)$ is a decreasing function of $u \in(0,+\infty)$. Therefore, (3.3) implies that

$$
\lim _{u \rightarrow+\infty} d\left(\phi_{u}(z), 1\right)=0
$$

\section{Proof of Theorem 3}

$(3) \Rightarrow(2)$ : Suppose that $h$ is conformal at 1 . Let $g_{2}$ be the Möbius transformation that maps the half-plane $H_{\rho}$ onto the unit disk so that $g_{2}(0)=0$ and $g_{2}(\infty)=1$. Then the function $g=g_{2} \circ h$ is a conformal map of $\mathbb{D}$ into itself and for its angular derivative at 1 we have $g^{\prime}(1) \in(0,+\infty)$. Let

$$
\Delta_{\eta}=\{z \in \mathbb{D}: d(z, 1)<\eta\}
$$

be a horodisk at 1. By Julia's lemma (see e.g. [1, §1.2.1]), the image of $\Delta_{\eta}$ under $g$ is contained in the horodisk $\Delta_{\eta g^{\prime}(1)}$. It follows that $h\left(\Delta_{\eta}\right)$ lies in the half-plane $g_{2}^{-1}\left(\Delta_{\eta g^{\prime}(1)}\right)$ which is strictly contained in $H_{\rho}$.

$(2) \Rightarrow(1)$ : Let $\Delta_{\eta}, \eta>0$, be a horodisk at 1 . Because of $(2), h\left(\Delta_{\eta}\right)$ lies in a horizontal half-plane

$$
H_{\rho^{\prime}}=\left\{\zeta: \operatorname{Im} \zeta>-\rho^{\prime}\right\}
$$

with $\rho^{\prime}<\rho$. We choose a $w \in \Omega \backslash H_{\rho^{\prime}}$; note that $\Omega \backslash H_{\rho^{\prime}} \neq \varnothing$ because $H_{\rho}$ is the smallest horizontal half-plane containing $\Omega$. Set $z:=h^{-1}(w)$ and consider the trajectory $\gamma_{z}$ starting from $z$. Since $h\left(\gamma_{z}\right)=\{w+t: t \geq 0\}$ lies outside $h\left(\Delta_{\eta}\right)$, we infer that $\gamma_{z}$ lies outside the horodisk $\Delta_{\eta}$. This means that the semigroup $\left(\phi_{t}\right)$ is of finite shift.

$(1) \Rightarrow(3)$ : Suppose that the semigroup $\left(\phi_{t}\right)$ is of finite shift. Consider the member function $\phi_{1}$ of the semigroup. By Corollary 2, $\phi_{1}$ is of finite shift. Let $\sigma_{1}: \mathbb{D} \rightarrow \mathbb{C}$ 
be the Koenigs function associated with $\phi_{1}$ and a point $z_{o} \in \mathbb{D}$. This is a function that satisfies in $\mathbb{D}$ the Abel equation

$$
\sigma_{1} \circ \phi_{1}=\sigma_{1}+1
$$

and it is obtained as a limit of a specific sequence of functions; see [8, Theorem 2.2]. We may assume that $\sigma_{1}^{\prime}\left(z_{o}\right) \neq 0$; see [8, Remark 3.2]. By [8, Proposition 3.3], $\sigma_{1}(\mathbb{D})$ lies in a horizontal half-plane. By composing by a Möbius transformation, we may assume that this half-plane is $H_{\rho}$. Observe that by (1.4), $h$ satisfies the Abel equation

$$
h \circ \phi_{1}=h+1 .
$$

By a theorem of Poggi-Corradini ([8, Theorem 3.4] which is a special case of [18, Proposition 3.1]), there exists a holomorphic function $F: H_{\rho} \rightarrow H_{\rho}$ such that

$$
h=F \circ \sigma_{1} \quad \text { and } \quad F(w+1)=F(w)+1, w \in H_{\rho} .
$$

We will show that $F$ is conformal at $\infty$. Note first that $F$ has no interior fixed point. Indeed, suppose that $w_{o} \in H_{\rho}$ is a fixed point of $F$. Then, by (4.1),

$$
F\left(w_{o}+1\right)=F\left(w_{o}\right)+1=w_{o}+1 .
$$

So $w_{o}+1$ is an interior fixed point too. Contradiction, because a holomorphic selfmap of $H_{\rho}$ can have at most one interior fixed point. We infer that the Denjoy-Wolff point $w^{*}$ of $F$ lies on the boundary of $H_{\rho}$. Suppose that $w^{*} \in \partial H_{\rho} \cap \mathbb{C}$. Then, by the Denjoy-Wolff theorem,

$$
\lim _{n \rightarrow+\infty} F_{n}(w)=w^{*}, \quad w \in H_{\rho} .
$$

It follows that for $w \in H_{\rho}$,

$$
\begin{aligned}
\lim _{n \rightarrow+\infty} F_{n}(w+1) & =\lim _{n \rightarrow+\infty} F_{n-1} \circ F(w+1) \\
& =\lim _{n \rightarrow+\infty} F_{n-1}(F(w)+1) \\
& =\lim _{n \rightarrow+\infty} F_{n}(w)+1=w^{*}+1,
\end{aligned}
$$

contradicting (4.3). Hence the Denjoy-Wolff point of $F$ is $\infty$. By the Denjoy-Wolff theorem (see e.g. [22, p. 78]), $F$ has finite angular derivative at $\infty$ and $F^{\prime}(\infty) \geq 1$.

Let $\psi$ be a Möbius transformation that maps $H_{\rho}$ conformally onto $\mathbb{D}$ with $\psi(\infty)=1$. Recall that $h=F \circ \sigma_{1}: \mathbb{D} \rightarrow H_{\rho}$. We have just proved that $F$ is conformal at $\infty$. This means that the function

$$
\psi \circ F \circ \psi^{-1}: \mathbb{D} \rightarrow \mathbb{D}
$$

is conformal at 1 . The Koenigs function $\sigma_{1}$ is also conformal at 1 by [10, Theorem 4.1]. Hence the function

$$
\psi \circ \sigma_{1}: \mathbb{D} \rightarrow \mathbb{D}
$$

is conformal at 1. It follows (see [7, Lemma 2]) that the function

$$
\psi \circ h=\psi \circ F \circ \sigma_{1}=\left(\psi \circ F \circ \psi^{-1}\right) \circ\left(\psi \circ \sigma_{1}\right): \mathbb{D} \rightarrow \mathbb{D}
$$

is conformal at 1 . Therefore, $h$ is conformal at 1 . 


\section{REFERENCES}

[1] Marco Abate, Iteration theory of holomorphic maps on taut manifolds, Research and Lecture Notes in Mathematics. Complex Analysis and Geometry, Mediterranean Press, Rende, 1989. MR.1098711 (92i:32032)

[2] I. N. Baker and Ch. Pommerenke, On the iteration of analytic functions in a halfplane. II, J. London Math. Soc. (2) 20 (1979), no. 2, 255-258, DOI 10.1112/jlms/s2-20.2.255. MR.551452 (83j:30024)

[3] D. Betsakos, On the asymptotic behavior of the trajectories of semigroups of holomorphic functions, Preprint 2014. To appear in the J. Geom. Anal.

[4] Manuel D. Contreras and Santiago Díaz-Madrigal, Analytic flows on the unit disk: angular derivatives and boundary fixed points, Pacific J. Math. 222 (2005), no. 2, 253-286, DOI 10.2140/pjm.2005.222.253. MR2225072 (2007a:30005)

[5] Manuel D. Contreras, Santiago Díaz-Madrigal, and Pavel Gumenyuk, Loewner chains in the unit disk, Rev. Mat. Iberoam. 26 (2010), no. 3, 975-1012, DOI 10.4171/RMI/624. MR2789373(2012a:30052)

[6] Manuel D. Contreras, Santiago Díaz-Madrigal, and Pavel Gumenyuk, Slope Problem for Trajectories of Holomorphic Semigroups in the Unit Disk, Comput. Methods Funct. Theory 15 (2015), no. 1, 117-124, DOI 10.1007/s40315-014-0092-9. MR3318309

[7] M. D. Contreras, S. Díaz Madrigal, and Ch. Pommerenke, On boundary critical points for semigroups of analytic functions, Math. Scand. 98 (2006), no. 1, 125-142. MR2221548 (2007b:30026)

[8] Manuel D. Contreras, Santiago Díaz-Madrigal, and Christian Pommerenke, Some remarks on the Abel equation in the unit disk, J. Lond. Math. Soc. (2) 75 (2007), no. 3, 623-634, DOI 10.1112/jlms/jdm013. MR2352725 (2008i:30025)

[9] Manuel D. Contreras, Santiago Díaz-Madrigal, and Christian Pommerenke, Iteration in the unit disk: the parabolic zoo, Complex and harmonic analysis, DEStech Publ., Inc., Lancaster, PA, 2007, pp. 63-91. MR2387282 (2009d:30062)

[10] Manuel D. Contreras, Santiago Díaz-Madrigal, and Christian Pommerenke, Second angular derivatives and parabolic iteration in the unit disk, Trans. Amer. Math. Soc. 362 (2010), no. 1, 357-388, DOI 10.1090/S0002-9947-09-04873-9. MR2550155 (2010j:30051)

[11] Carl C. Cowen, Iteration and the solution of functional equations for functions analytic in the unit disk, Trans. Amer. Math. Soc. 265 (1981), no. 1, 69-95, DOI 10.2307/1998482. MR607108(82i:30036)

[12] Mark Elin, Fiana Jacobzon, Marina Levenshtein, and David Shoikhet, The Schwarz lemma: rigidity and dynamics, Harmonic and complex analysis and its applications, Trends Math., Birkhäuser/Springer, Cham, 2014, pp. 135-230, DOI 10.1007/978-3-319-01806-5_3. MR.3203101

[13] Mark Elin and David Shoikhet, Linearization models for complex dynamical systems, Topics in univalent functions, functional equations and semigroup theory; Linear Operators and Linear Systems, Operator Theory: Advances and Applications, vol. 208, Birkhäuser Verlag, Basel, 2010. MR2683159 (2012c:30042)

[14] John B. Garnett and Donald E. Marshall, Harmonic measure, New Mathematical Monographs, vol. 2, Cambridge University Press, Cambridge, 2005. MR2150803 (2006g:31002)

[15] V. V. Goryaĭnov, Semigroups of analytic functions in analysis and applications (Russian, with Russian summary), Uspekhi Mat. Nauk 67 (2012), no. 6(408), 5-52, DOI 10.1070/RM2012v067n06ABEH004816; English transl., Russian Math. Surveys 67 (2012), no. 6, 975-1021. MR 3075076

[16] Fiana Jacobzon, Marina Levenshtein, and Simeon Reich, Convergence characteristics of one-parameter continuous semigroups, Anal. Math. Phys. 1 (2011), no. 4, 311-335, DOI 10.1007/s13324-011-0021-2. MR:2887103 (2012m:37083)

[17] Pietro Poggi-Corradini, Backward-iteration sequences with bounded hyperbolic steps for analytic self-maps of the disk, Rev. Mat. Iberoamericana 19 (2003), no. 3, 943-970, DOI 10.4171/RMI/375. MR2053569 (2006f:30025)

[18] Pietro Poggi-Corradini, On the uniqueness of classical semiconjugations for self-maps of the disk, Comput. Methods Funct. Theory 6 (2006), no. 2, 403-421, DOI 10.1007/BF03321619. MR2291141(2007j:30017) 
[19] Pietro Poggi-Corradini, Pointwise convergence on the boundary in the Denjoy-Wolff theorem, Rocky Mountain J. Math. 40 (2010), no. 4, 1275-1288, DOI 10.1216/RMJ-2010-40-4-1275. MR:2718814 (2011h:30040)

[20] Ch. Pommerenke, On the iteration of analytic functions in a halfplane, J. London Math. Soc. (2) 19 (1979), no. 3, 439-447, DOI 10.1112/jlms/s2-19.3.439. MR540058 (83j:30023)

[21] Ch. Pommerenke, Boundary behaviour of conformal maps, Grundlehren der Mathematischen Wissenschaften [Fundamental Principles of Mathematical Sciences], vol. 299, Springer-Verlag, Berlin, 1992. MR,1217706 (95b:30008)

[22] Joel H. Shapiro, Composition operators and classical function theory, Universitext: Tracts in Mathematics, Springer-Verlag, New York, 1993. MR1237406 (94k:47049)

Department of Mathematics, Aristotle University of Thessaloniki, 54124 ThessaLONIKI, GREECE

E-mail address: betsakos@math.auth.gr 